\title{
Weltmärkte im Wandel - Nutzt Europa seine Chancen?
}

Die Handelspolitik ist wieder zurück. Sie spielte lange Zeit keine große Rolle in der wirtschaftspolitischen Diskussion. Wir befinden uns jetzt jedoch in einer Phase der Neuorientierung auf dem Weltmarkt. Die multilateralen Handelsabkommen werden ausgehend von kritischen Stimmen aus der Bevölkerung bis hin zu Entscheidungsträgern infrage gestellt. Mit dem Resultat, dass zunehmend bilaterale Abkommen eine Rolle spielen und sich die Handelsströme verändern. Das heißt aber auch, dass wenn Gruppen sich neu zusammenschließen, andere draußen bleiben. Dabei ist die Rolle der Welthandelsorganisation (WTO) als moderierender Organisator des Welthandels deutlich schwächer geworden. Auch die EU reagiert mehr, als dass sie agiert. Auf der diesem Heft zugrundeliegenden Konferenz „Weltmärkte im Wandel - Nutzt Europa seine Chancen?", die der Wirtschaftsdienst gemeinsam mit dem ifo Institut am 5. Dezember 2017 in Berlin veranstaltet hat, wurden diese Themen adressiert.

Die Globalisierung und damit die weitere Vertiefung der wirtschaftlichen Integration werden von verschiedenen Seiten infrage gestellt, betonte Clemens Fuest, der Präsident des ifo Instituts, in seiner Keynote zu „Steuerpolitik als Mittel des Protektionismus?". In vielen Ländern sind protektionistische Tendenzen zu erkennen. Die US-Steuerreform enthält ebenfalls protektionistische Elemente, und es bestehen Anreize, einen Großteil der Gewinne in den USA zu versteuern. Damit würden wohl die größten Verlierer der Reform die Steueroasen sein. Die US-Steuerreform enthält zudem viele nicht abgestimmte Maßnahmen, die mit den Besteuerungsansprüchen anderer Länder kollidieren. Dieses Verhalten gilt allerdings nicht nur für die USA. Viele Länder verhalten sich zunehmend unkoordiniert, wodurch die grenzüberschreitende wirtschaftliche Aktivität behindert, die Investitionen verzerrt und die Steuerlast unsystematisch verteilt wird.

Die erste Session der Konferenz fragte nach der Entschleunigung im Welthandel. Holger Breinlich von der Nottingham University behandelt das Thema: „Dienstleistungshandel: historische und aktuelle Perspektiven“. Der internationale Dienstleistungshandel spielt eine immer bedeutendere Rolle im Welthandel. Er entspricht etwa einem Viertel des gesamten Welthandels. Hierbei muss im Gegensatz zu Zollschranken im Warenhandel auf die nicht-tarifären Handelshemmnisse geschaut wer- den, die oft nur schwer quantifizierbar sind. Zum besseren Verständnis des Dienstleistungshandels hat die volkswirtschaftliche Forschung in jüngster Zeit viel beigetragen. Diese Ergebnisse sollten bei den anstehenden Herausforderungen wie der Herauslösung Großbritanniens aus der Europäischen Union (Brexit) und der protektionistischen Handelspolitik der USA berücksichtigt werden.

Ralph Ossa von der Universität Zürich widmet sich in seinem Beitrag der Frage: „Wie teuer wäre ein Handelskrieg?". Zurzeit sprechen bereits viele von einer drohenden Entwicklung in diese Richtung. Daher ist es sinnvoll zu hinterfragen, was schlimmstenfalls passieren kann. Die einfache Botschaft lautet, dass Gewinne aus dem Handel hoch und Handelskriege teuer sind. So ist etwa ein Viertel des weltweiten Realeinkommens auf Handel zurückzuführen. Ein Handelskrieg würde fast ein Viertel dieser Handelsgewinne zerstören und erheblichen Schaden anrichten.

Alexander Keck von der WTO zeigt gemeinsam mit seinen Kollegen John Hancock und Coleman Nee die Perspektiven für den Welthandel und das internationale Handelssystem auf. Sie betonen, dass der Handel trotz des jüngsten Rückgangs für Wachstum und Entwicklung wichtig ist. Eine Herausforderung der weiter fortschreitenden weltweiten ökonomischen Integration liegt in der schnellen Veränderung des Handels selbst und der Wirkung der verbleibenden Handelsschranken. Die Autoren heben neue Modelle der Handelskooperation hervor, die dazu beitragen können, auf globaler Ebene Fortschritte $z u$ erzielen und gleichzeitig den unterschiedlichen Interessen und Entwicklungsniveaus der Länder Rechnung zu tragen.

Die zweite Session der Konferenz identifiziert den Handlungsbedarf in der europäischen Außenwirtschaftspolitik. Den Anfang machen Guntram B. Wolff von der wirtschaftswissenschaftlichen Denkfabrik Bruegel und seine Kollegin Maria Demertzis sowie André Sapir vom European Center for Advanced Research in Economics and Statistics (ECARES) der Freien Universität Brüssel. Sie betrachten Europa in einer neuen Weltordnung. Die EU stellt eine relativ offene Volkswirtschaft dar und hat vom multilateralen System profitiert. Daher sollte sie ihre strategischen Interessen verteidigen. Das Singapur-Urteil, 
das besagt, dass die EU nicht ohne die Zustimmung der nationalen Parlamente Handelsabkommen abschließen darf, hat hier eine nützliche Klarstellung zur Handelspolitik geboten. Die Beseitigung interner Ungleichgewichte würde auch die externe Glaubwürdigkeit der EU in der Handelspolitik erhöhen. Schließlich würde die Stärkung des europäischen Sozialmodells ein Gegenmodell zu protektionistischen Versuchungen darstellen.

Gabriel Felbermayr vom ifo Zentrum Außenwirtschaft des ifo Instituts stellt in seinem Beitrag „EU-Handels- und Investitionspolitik" den aktuellen Forschungsstand zu Freihandelsabkommen, Handelsschutzmaßnahmen und ausländischen Investitionen vor. In der EU-Handels- und Investitionspolitik gibt es viele Baustellen. Um die bestehenden und geplanten Freihandelsabkommen beurteilen zu können, werden die einzelnen Effekte detailliert aufgezeigt. Die Wirkungen der europäischen Handelspolitik sowie des neuen Protektionismus der USA werden untersucht und erläutert.

Dennis Novy fragt gemeinsam mit seinen Kollegen Sascha O. Becker und Thiemo Fetzer vom Department of Economics und dem Centre for Competitive Advantage in the Global Economy (CAGE) der Universität Warwick: „Welche Abstimmungsmuster liegen dem Brexit-Referendum zugrunde?". Das Brexit-Referendum vom 23. Juni 2016 stellt einen zentralen Wendepunkt in der britischen Nachkriegsgeschichte dar. Bei der Analyse der Wahlergebnisse zeigt sich, dass das Abstimmungsverhalten für den EU-Austritt offenbar wenig mit der Handels- und Wirtschaftspolitik der EU zu tun hatte. Stattdessen ist die Furcht vor Einwanderung, insbesondere in Gebieten, in denen diese kaum auftritt, entscheidender. Unzufriedenheit mit den allgemeinen wirtschaftlichen Zuständen und dem öffentlichen Versorgungsangebot spielen auch eine wesentliche Rolle.

Die dritte Session fragt nach den Herausforderungen für Wirtschaft und Politik. Dalia Marin von der Ludwig-Maximilians-Universität in München hat zum Thema „Globale Wertschöpfungsketten, der Aufstieg der Roboter und das Humankapital" referiert. Oft wird argumentiert, dass der Einsatz von Robotern dazu führt, dass die Produktionsprozesse von den Niedriglohnländern in reiche Länder zurückgeholt werden - also eine Wiederbelebung der Produktion in reichen Ländern stattfindet. Aber die intelligenten Roboter sind eine kapitalintensive Technologie, die intelligente Menschen potenziell ersetzen kann. Infolgedessen ist die Qualifikationsprämie - der relative Lohn zwischen einem Hochschulabsolventen und einem Abiturienten - in Europa rückläufig. Der Ausbau der Hochschulbildung war angesichts der Kapitalverzerrung der Digitaltechnologie zu aggressiv.
Dr. Brigitte Preissl ist Chefredakteurin von Wirtschaftsdienst und Intereconomics und leitet den Bereich Wissenstransfer Wirtschaftswissenschaften der ZBW Leibniz-Informationszentrum Wirtschaft in Hamburg.

Kristin Biesenbender, M.A., und Cora WackerTheodorakopoulos, Dipl.-Volkswirtin, sind dort Redakteurinnen.

Marc-Andreas Mündler von der University of California in San Diego setzt den Fokus seines Beitrags auf „Außenhandel, Arbeitsmärkte und die globale Verteilung der Einkommen“. Die Globalisierung schreitet voran und nimmt Einfluss auf die Einkommensverteilung zwischen Ländern und innerhalb von Ländern. Die Erforschung dieses Zusammenhangs hat sich weiterentwickelt und bietet verbesserte und nuanciertere Erklärungen als die frühen Ansätze von Adam Smith und David Ricardo. Doch deren grundlegende Einsichten in die Vorteile der globalen Arbeitsteilung bleiben unverändert relevant. Mit der Globalisierung 2.0 werden nicht mehr nur Endprodukte gehandelt. Die moderne Weltwirtschaft tauscht Komponenten, Zwischenprodukte und Dienstleistungen. Deren Produktionsstufen siedeln sich zumeist in qualifikationsärmeren Ländern an, was zu einer erhöhten Lohnungleichheit innerhalb der Länder führt. Jüngste Fortschritte in der internationalen Handelstheorie liefern ein weitergehendes Verständnis der Einflüsse auf die Einkommensverteilung, einerseits herbeigeführt durch Lohnunterschiede zwischen mehr und weniger globalisierten Unternehmen und andererseits verursacht durch die globalisierungsbedingte Arbeitsteilung zwischen Berufen innerhalb von Betrieben und die damit einhergehende Lohnspreizung.

Zum Thema der abschließenden Podiumsdiskussion „In welche Richtung verschieben sich die globalen Gewichte? Was bedeutet das für Europa?" hat Johannes Wallacher einen Aufsatz über „Ethische Maßstäbe für gerechte Regeln des Welthandels“ beigetragen. Internationale Abkommen sind in Verruf geraten. Die einen befürchten sinkende Sozial-, Umwelt- und Verbraucherschutzstandards und die anderen eine zu weitgehende Bevormundung. Dabei muss allerdings auch berücksichtigt werden, wie das Ergebnis aussieht, wenn überhaupt kein Abkommen entsteht. Das globale Gemeinwohl, Transparenz und Chancengleichheit auch für weniger entwickelte Länder sollten die vorrangigen Ziele sein. 